\title{
Comparing Simulation Use Cases in Building Design for Developing an User-centric Simulation Platform
}

\author{
Sean Hay Kim \\ Seoul National University of Science and Technology \\ Seoul, Korea \\ seanhay.kim@seoultech.ac.kr
}

\section{Extended Abstract}

Background and objective:

Building energy simulations not only evaluate the performance of a fixed design, but also work as a decision support tool that provides the designer the information and knowledge about how the design should be developed in order to achieve higher performance. Additionally it helps the designer acquiring energy characteristics and behaviour of building systems, the knowledge of which building designers tend to be disadvantageous to earn.

Despite the merit of simulations, it was due to its expensive cost that simulations have not been actively used in building and system design process. As more and more designers use BIM (Building Information Modelling) and automatic model transformations to performance models are available, however, use of simulations becomes a de facto standard design process among acknowledged designers.

While simulations are one of design tools for MEP (Mechanical Electrical and Plumbing) engineers in order to assess building cooling and heating loads and analyse the energy performance of the HVAC design in the United States, they are not widely used in Korea due to less localization, less experience and thus less trust over simulations. This study tries to identify what barriers and issues block a wider use of simulations, and also to analyse what R\&D efforts are needed to accelerate use of simulations in Korea.

Scope:

One of issues is that workflows of existing simulation tools are not quite aligned to domestic building and system design process. It is because almost all building energy simulation tools are developed in U.S. and European countries, thus the simulation workflows represent the design workflow in those countries; domestic users do not tend to follow up the presented information flows, do not use the presented assumptions, defaults and library, and even do not tend to understand the user interface of existing simulation tools beyond language problems. This finding suggests developing a new building energy simulation platform that meets domestic users' requirement and offers better design productivity. This study, therefore, results in the following artifacts.

First, this study investigates both domestic and international R\&D efforts in building simulation usability areas. Second, this study employs a survey to collect simulation users' opinions including architects, MEP designers, building energy consultants concerning how simulations are used in domestic building and system design process, what kinds of problems hinder the extension and how to improve usability. Third, this study benchmarks an IDP (Integrated Design Process) developed by Stantec, an U.S. engineering firm which is known enhancing collaboration between architects and engineers by means of using simulations as a communication tool. Lastly, this study suggests functional and usability improvements of simulations and protocols that would result in enhanced productivity and rational decision-making.

Result:

Since this research is still on-going, one of by-products is discussed in details; two simulation usecases during building and system design process - the IDP and the other observed in domestic design process - are compared and then significance and insights are discussed. In particular, the discussion focuses on LOD (Level of Detail) of simulations, which varies upon steps and stages of the building and system design process. Compared to the IDP, it is apparently observed that less or least domestic MEP engineers involve in early design phases where design innovations driven by simulation are mostly highlighted, thus LOD of HVAC systems tends to be decided at very late design phases (e.g., construction document phase). This implies new simulation platform should contain a function to evaluate the possibility of switching to totally different 
HVAC systems until very late design phases, rather than fixing them at early phases and then recurring it till the end of design process as most simulation tools assume.

\section{Acknowledgement}

This research was supported by Project to Establish Professional Graduate School on Design Engineering supervised by the Korea Institute of Design Promotion and sponsored by the Ministry of Trade, Industry and Energy (N0001436). This research was supported by Basic Science Research Program through the National Research Foundation of Korea (NRF) funded by the Ministry of Science, ICT \& Future Planning (NRF-2015R1C1A2A01053559). 